\title{
Reduced-Bandwidth Duobinary Differential Continuous-Phase Modulation Format for Optical Communications
}

\author{
David Boivin, Marc Hanna, and John R. Barry
}

\begin{abstract}
We demonstrate a new modulation format based on differential phase-shift keying (DPSK) with a duobinary-shaped modulating signal for applications in optical communications. Compared to conventional DPSK and using the 20-dB full-width criterion, numerical and experimental data show a bandwidth reduction by a factor of two resulting in a better tolerance to group-velocity dispersion but at the expense of a back-to-back sensitivity degradation of $3.5 \mathrm{~dB}$.
\end{abstract}

Index Terms-Differential phase-shift keying (DPSK), duobinary modulation, modulation formats, optical fiber communications.

\section{INTRODUCTION}

$\mathbf{R}$ ESEARCHERS constantly endeavour to reach the optical fiber capacity in the Shannon sense. Among different possible approaches, one consists of increasing the bit rate by employing modulation formats that possess a higher spectral efficiency [1], [2]. For a given bit rate, each channel occupies less bandwidth, thereby allowing more information to be conveyed in an equivalent wavelength range. In an intensity modulation context, duobinary modulation was shown to be an attractive candidate [3] because of its bandwidth compression. Recently, attention focused on phase-modulated formats as promising updates for future optical communication systems. If few studies proposed multilevel formats such as return-to-zero differential quadrature phase-shift keying (RZ-DQPSK) [4], most concentrated on binary differential phase-shift keying (DPSK) and its natural evolution toward RZ-DPSK [5]-[7] because RZ formats have been shown to be better suited for nonlinear transmission. Phase modulation possesses several advantages over intensity modulation in a wavelength-divisionmultiplexing (WDM) system: better tolerance to channel nonlinearities and cascaded filtering, and a 3-dB higher sensitivity. However, ON-OFF signalling has always been used in phase-modulated systems resulting in abrupt switchings in the time domain that translate into large spectral sidelobes outside of the main spectral band. In the radio-frequency domain, continuous phase-modulated (CPM) systems were shown to overcome these limitations [8]. In this letter, we present the first implementation of a partial response optical CPM system based

Manuscript received October 4, 2004; revised February 2, 2005.

D. Boivin and M. Hanna are with FEMTO-ST/GTL-CNRS Telecom, Metz 57070, France (e-mail: david.boivin@georgiatech-metz.fr).

J. R. Barry is with the School of Electrical and Computer Engineering, Georgia Institute of Technology, Atlanta, GA 30332-0250 USA

Digital Object Identifier 10.1109/LPT.2005.846572
TABLE I

EXAMPLE OF DUOBINARY DPSK ENCODING

\begin{tabular}{c|c|c||c|c|c|c|c|c}
\hline Data & $\mathrm{x}$ & $\mathrm{x}$ & $\mathbf{1}$ & $\mathbf{1}$ & $\mathbf{1}$ & $\mathbf{0}$ & $\mathbf{1}$ & $\mathbf{1}$ \\
\hline \hline \multicolumn{1}{c}{ Differential precoding } \\
\hline Duobinary signal \\
\hline DPSK $p_{1}[k]$ & $\mathrm{x}$ & 0 & 1 & 0 & 1 & 1 & 0 & $\mathbf{1}$ \\
\hline Duobinary $p_{2}[k]$ & 0 & 0 & 1 & 1 & 0 & 1 & 1 & 0 \\
\hline \hline & 0 & 0 & 1 & 2 & 1 & 1 & 2 & 1 \\
\hline$s_{\text {duo }}[k]$ & 0 & MZDI \\
\hline \hline Constructive port & 0 & 1 & 0 & 0 & 0 & 1 & 0 & 0 \\
\hline Destructive port & 1 & 0 & $\mathbf{1}$ & $\mathbf{1}$ & $\mathbf{1}$ & $\mathbf{0}$ & $\mathbf{1}$ & $\mathbf{1}$ \\
\hline
\end{tabular}

on a duobinary phase response and show its reduced bandwidth occupation and tolerance to group-velocity dispersion (GVD) by comparison with conventional DPSK. This narrowing of the spectrum is at the expense of a measured back-to-back sensitivity degradation of $3.5 \mathrm{~dB}$.

\section{PRINCIPLE}

Table I shows a practical example of duobinary DPSK modulation. For DPSK-based modulation, the data stream $s_{\text {data }}[k]$ at rate $R$ has to be differentially encoded. This logical XOR operation results in the first precoded sequence $p_{1}[k]=s_{\text {data }}[k] \oplus$ $p_{1}[k-1]$. The generation of the duobinary signal requires an additional precoding step, which is identical to the first one, yielding the output sequence

$$
p_{2}[k]=p_{1}[k] \oplus p_{2}[k-1] .
$$

This signal is passed through a fifth-order Bessel lowpass filter with a $0.28 R$ cutoff frequency. Binary precoded data $p_{2}[k]$ are thereby transformed into a three-level duobinary signal [3]: $s_{\text {duo }}[k]=p_{2}[k]+p_{2}[k-1]$.

The operation that converts $p_{1}[k]$ into $s_{\text {duo }}[k]$ has the following one-to-one mapping property: a logical " 1 " in $p_{1}[k]$ results in a " 1 " in $s_{\text {duo }}[k]$, while a " 0 " in $p_{1}[k]$ results in a " 0 " or " 2 " in $s_{\text {duo }}[k]$ depending on the memory introduced by the duobinary filtering.

When the phase modulator is driven by a $2 V_{\pi}$ amplitude duobinary signal, the optical phase has continuous variations between $0, \pi$, and $2 \pi$. As in intensity duobinary modulation, logical levels are well defined only at the center of the bit slots, and large oscillations occur between two successive sampling times. Allowing these oscillations is at the origin of the reduced spectral width. 
Binary precoded data : $\mathbf{p}_{2}(\mathrm{t})$
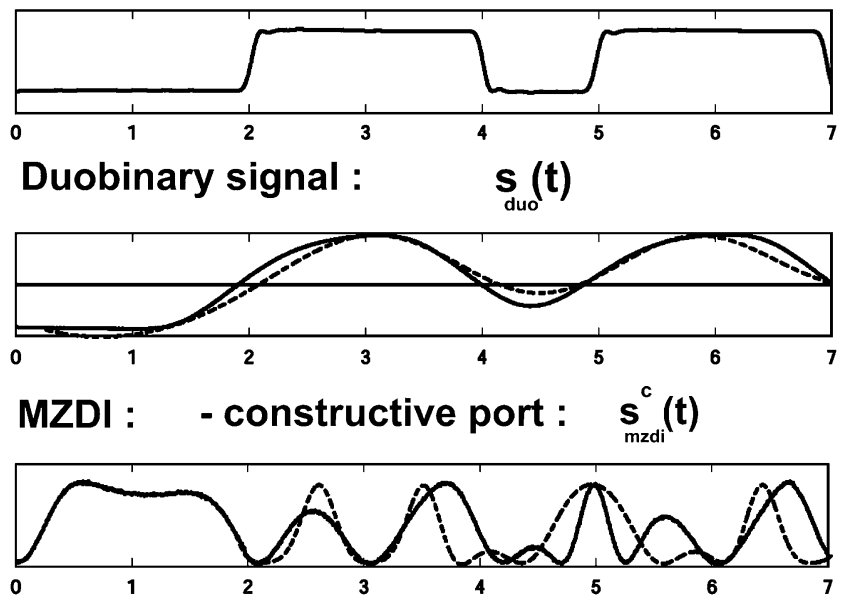

- destructive port : $\quad s_{\text {mzdi }}^{d}(t)$

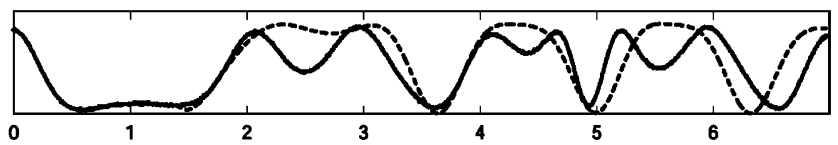

Fig. 1. Experimental signals corresponding to the data sequence of Table I.

Because photodetection is phase insensitive, a phase-to-intensity conversion is needed at the receiver. The optical signal is, therefore, demodulated by a Mach-Zehnder delay interferometer (MZDI) that performs the interference between two successive bits. It is clear that removing the first two bits of MZDI sequences due to the two precodings, we recover $\overline{s_{\text {data }}[k]}$ (respectively, $s_{\text {data }}[k]$ ) at the MZDI constructive (respectively, destructive) port. This is illustated in Fig. 1, which shows the electrical and optical signals at different points in the system. The simulations (dashed lines) are obtained by use of an ideal duobinary filter $(h(\omega)=\cos (\omega T / 2))$ that produces a slightly different signal compared to the experimental Bessel filter (solid line). Combined with demodulator imperfections, it explains the mismatch in the waveforms although logical values are equal at sampling times. As in a standard DPSK system, balanced detection can be used to obtain a $3-\mathrm{dB}$ increase in sensitivity.

\section{RESULTS AND DISCUSSION}

The experimental setup is depicted in Fig. 2. A distributed feedback laser feeds a $10-\mathrm{Gb} / \mathrm{s}$ phase modulator with $V_{\pi}=$ $5.8 \mathrm{~V}$. This modulator is driven either directly with a $2-\mathrm{Gb} / \mathrm{s}$ pseudorandom bit sequence with amplitude $V_{\pi}$ for DPSK or with the corresponding duobinary signal with amplitude $2 V_{\pi}$ for duobinary DPSK. A scanning Fabry-Pérot interferometer is used to measure the spectra of the output signals. The MZDI was fabricated from two fiber couplers and thermally insulated.

Fig. 3 shows the measured eye diagram at the output of the constructive port. The large oscillations between sampling times in the duobinary case are clearly observed. The asymmetry in the observed space level is due to imperfections in the experimental setup. The simulated eye diagram [Fig. 4(b)] does not exhibit such an asymmetry.

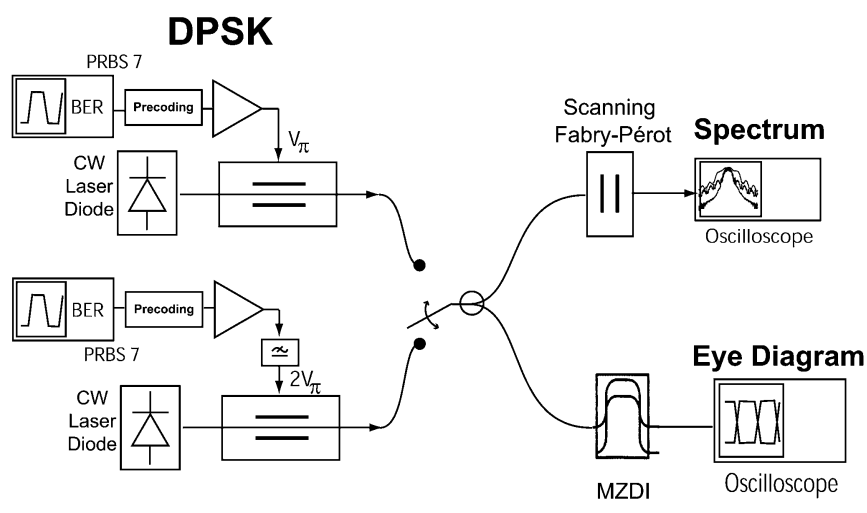

\section{Duobinary DPSK}

Fig. 2. Experimental setup.

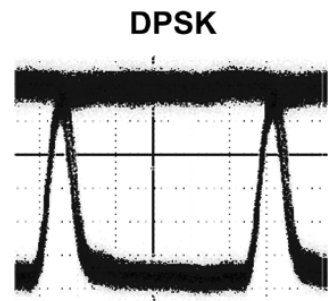

duobinary DPSK

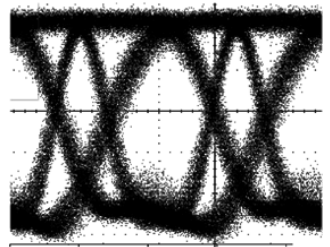

Fig. 3. Experimental eye diagrams for DPSK and duobinary DPSK signals. a)

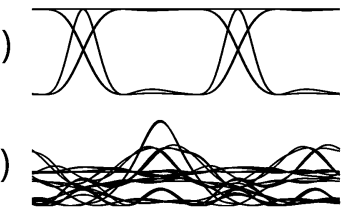

b) duobinary DPSK

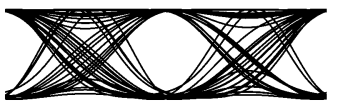

d)

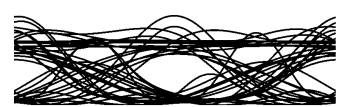

Fig. 4. GVD tolerance for DPSK and duobinary DPSK. (a)-(b) Back-to-back. (c)-(d) After $40 \mathrm{~km}$ of SMF.

The DPSK eye diagram also shows sharp rises between two successive bit slots. This is easily explained by the use of a finite-bandwidth phase modulator that does not produce perfect $\pi$ phase jumps, as opposed to the intensity modulator technique usually used to generate the optical DPSK signals [5].

Fig. 5 shows the bit-error rate as a function of optical power for duobinary DPSK and DPSK in a back-to-back configuration. For this purpose, an optical attenuator was inserted between the emitter and the error detector. A $2.6-\mathrm{GHz}$ electrical lowpass filter was used at the detection. A thermal noise sensitivity degradation of $3.5 \mathrm{~dB}$ is observed for duobinary DPSK. This is attributed to the eye opening penalty due to oscillations between sampling times and imperfections in the MZDI and duobinary filter mentioned earlier. In the context of intensity modulation, this penalty was also observed for phase-shaped binary transmission [9] when compared to nonreturn-to-zero and evaluated around $3 \mathrm{~dB}$.

Fig. 6 shows the optical spectra for DPSK and duobinary DPSK signals with respect to the optical frequency normalized to the bit rate. The superiority of duobinary DPSK over DPSK modulation in terms of bandwidth requirements is clearly 


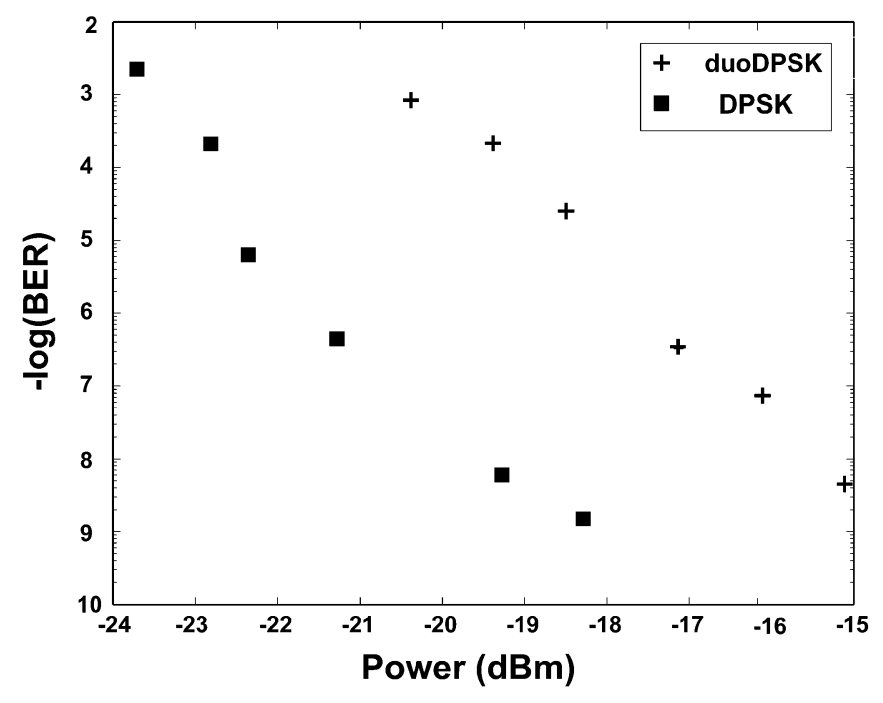

Fig. 5. Experimental back-to-back sensitivity.

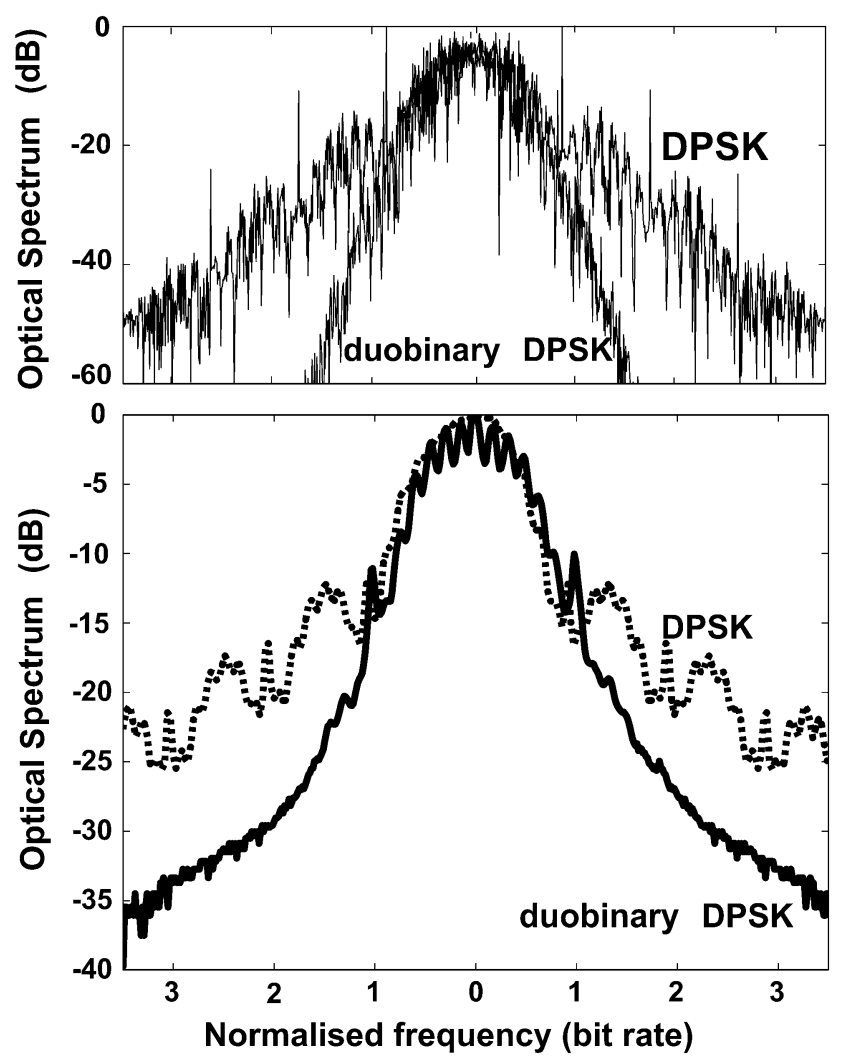

Fig. 6. Optical spectra of DPSK and duobinary DPSK signals, with frequency axis normalized to the bit rate (simulations: top; experiment: bottom).

observed. Although the main lobes are of comparable width, the duobinary DPSK spectrum does not exhibit sidelobes. We, therefore, anticipate a better behavior against cascaded optical filtering used in a WDM environment. The spectrum is reduced by a factor of two if we use the 20-dB full-width criterion. If no filters are used, this reduction results in a better tolerance to GVD. The 10-Gb/s simulations shown in Fig. 4 demonstrate a lower eye-opening penalty for duobinary DPSK after $40-\mathrm{km}$ propagation in standard single-mode fiber with dispersion parameter $D=17 \mathrm{ps} /(\mathrm{nm} \cdot \mathrm{km})$.

\section{CONCLUSION}

We have reported the implementation in optics of a continuous-phase modulation format, duobinary DPSK, which substantially reduces the bandwidth occupation compared to standard DPSK and demonstrates a better tolerance against GVD. This is a first attempt to transpose the class of partial response CPM formats to the field of optical communications. These formats are well known for their robustness to nonlinearities and spectral efficiency in wireless applications, and are promising for future developments in WDM systems.

\section{REFERENCES}

[1] N. Yoshikane and I. Morita, "160\% spectrally-efficient $5.12 \mathrm{~Tb} / \mathrm{s}$ $(64 \times 85.4 \mathrm{~Gb} / \mathrm{s})$ RZ DQPSK transmission without polarization demultiplexing," in Proc. ECOC 2004, Stockholm, Sweden, Paper Th4.4.3.

[2] B. Zhu, L. Nelson, A. Gnauck, C. Doerr, J. Leuthold, L. Gruner-Nielsen, M. Pedersen, J. Kim, and R. L. Lingle Jr., "High spectral density long-haul 40-Gb/s transmission using CSRZ-DPSK format," J. Lightw. Technol., vol. 22, no. 1, pp. 208-214, Jan. 2004.

[3] T. Ono, Y. Yano, K. Fukuchi, T. Ito, H. Yamazaki, M. Yamaguchi, and K. Emura, "Characteristics of optical duobinary signals in terabit/s capacity, high spectral efficiency WDM systems," J. Lightw. Technol., vol. 16, no. 5, pp. 788-797, May 1998.

[4] P. S. Cho, V. S. Grigoryan, Y. A. Godin, A. Salamon, and Y. Achiam, "Transmission of 25-Gb/s RZ-DQPSK signals with $25-\mathrm{GHz}$ channel spacing over $1000 \mathrm{~km}$ of SMF-28 fiber," IEEE Photon. Technol. Lett., vol. 15, no. 3, pp. 473-475, Mar. 2003.

[5] J. H. Sinsky, A. Adamiecki, A. Gnauck, C. A. Burrus, J. Leuthold, O. Wohlgemuth, S. Chandrasekhar, and A. Umbach, "RZ-DPSK transmission using a 42.7-Gb/s integrated balanced optical front end with record sensivity," J. Lightw. Technol., vol. 22, no. 1, pp. 180-185, Jan. 2004.

[6] C. Rasmussen, T. Fjelde, J. Bennike, F. Liu, S. Dey, B. Mikkelsen, P. Mamyshev, P. Serbe, P. van der Wagt, Y. Akasaka, D. Harris, D. Gapontsev, V. Ivshin, and P. Reeves-Hall, "DWDM 40G transmission over trans-pacific distance $(10000 \mathrm{~km})$ using CSRZ-DPSK, enhanced FEC, and All-Raman-Amplified $100 \mathrm{~km}$ ultraWave fiber spans," $J$. Lightw. Technol., vol. 22, no. 1, pp. 203-207, Jan. 2004.

[7] T. Tsuritani, K. Ishida, A. Agata, K. Shimomura, I. Morita, T. Tokura, H. Taga, T. Mizuochi, N. Edagawa, and S. Akiba, "70-GHz-spaced $40 \times 42.7 \mathrm{~Gb} / \mathrm{s}$ transpacific transmission over $9400 \mathrm{~km}$ using prefiltered CSRZ-DPSK signals, all raman repeaters, and symmetrically dispersion-managed fiber spans," J. Lightw. Technol., vol. 22, no. 1, pp. 215-224, Jan. 2004.

[8] J. B. Anderson, T. Aulin, and C.-E. Sundberg, Digital Phase Modulation. New York: Plenum Press, 1986.

[9] S. Bigo, G. Charlet, and E. Corbel, "What has hybrid phase/intensity encoding brought to $40 \mathrm{Gbit} / \mathrm{s}$ ultralong-haul systems ?," in Proc. ECOC 2004, Stockholm, Sweden, Paper Th2.5.1. 